\title{
Influence de l'aménagement marémoteur de la Rance sur le milieu de sédimentation estuarien.
}

\section{Chantal BONNOT-COURTOIS}

URA 904 du CNRS. Dynamique et Gestion des Espaces Littoraux. Laboratoire

de Géomorphologie EPHE. Dinard.

\section{Bruno CALINE}

Sédimentologie ELF Aquitaine. Pau.

Robert MATHIEU, Rafika CHAIT, Marialsira FERNANDEZ

Laboratoire de Micropaléontologie. Université Fierre et Marie Curie. Paris VI. Jelle BEKS

N.1.O.Z. Netherlands Institute for Sea Research. Texel. The Netherlands.

Résumé

L'estuaire de la Rance est un exemple unique d'implantation d'une usine marémotrice mise en service il y a trente ans dans un secteur à très fortes marées et sur un fleuve à faible débit. La construction du barrage puis l'exploitation de l'usine ont entrainé des modifications hydrologiques qui ont eu des conséquences sur la sédimentation et l'environnement du milieu estuarien. Les premiers résultats des analyses sédimentologiques et micropaléontologiques, ainsi que des essais de datation sur quelques carottes prélevées à l'intérieur et à l'extérieur du bassin de retenue mettent en évidence les changements irtervenus dans les conditions de sédimentation des vases estuariennes.

\section{Introduction}

L'estuaire de la Rance est situé dans l'une des régions du monde où l'amplitude de la marée est la plus forte puisque le marnage atteint $13,60 \mathrm{~m}$ en période de vive-eau. L'usine marémotrice, construite au cours des années 1960 , est formée $d^{3}$ un ouvrage de $750 \mathrm{~m}$ de long qui isole un bassin de retenue d'une superficie de $22 \mathrm{~km}^{2}$, constituant un réservoir de 184 millions de $\mathrm{m}^{3}$. Pendant la construction du barrage, l'estuaire a été isolé du milieu marin pendant une période de 3 ans entre 1963 et 1966, puis la remise en communication avec la mer ouverte s'est effectuée depuis 1967.

L'exploitation de l'usine a entrainé des modifications du régime hydrologique de l'estuaire, essentiellement par prolongation des étales qui peuvent durer deux heures à l'intérieur du bassin au lieu d'une dizaine de minutes côté mer, et par 
réduction du marnage qui n'est plus que d'environ $9 \mathrm{~m}$ dans le bassin de retenue. Ces modifications hydrologiques ont des conséquences sur l'équilibre sédimentologique de l'estuaire. La prolongation des étales dans le bassin par rapport à la mer ouverte laisse aux particules en suspension le temps d'atteindre les fonds et d'y sédimenter. La réduction du marnage et en particulier le fait que le niveau du bassin ne descende que très exceptionnellement en-dessous de $4 \mathrm{~m}$ entraîne une diminution des courants de jusant qui sont moins efficaces pour éroder les fonds et évacuer vers l'extérieur les matières en suspension. L'annulation du courant pendant les étales prolongées et l'atténuation du jusant favorisent théoriquement la sédimentation, particulièrement dans les zones abritées des anses, sur les berges de l'estuaire ainsi que dans les secteurs calmes à l'amont du bassin maritime.

L'objectif de cette étude est de déterminer I'influence de l'implantation de l'usine marémotrice sur les conditions de sédimentation du milieu estuarien, d'une part par l'analyse de la répartition des sédiments superficiels dans le bassin et la comparaison avec les données antérieures, d'autre part à l'aide de carottages réalisés en divers points de l'estuaire et dans un estuaire voisin, et analysés du point de vue sédimentologique et microfaunistique. Des résultats préliminaires de datation par la méthode du ${ }^{210} \mathrm{~Pb}$ permettent d'estimer l'évolution des taux de sédimentation.

\section{Répartition des sédiments superficiels dans le bassin maritime.}

Les études réalisées dans les années 1950 pour l'implantation de l'usine marémotrice de la Rance concernaient principalement les paramètres hydrodynamiques ou la résistance et la corrosion des matériaux de l'ouvrage vis-à-vis de l'eau de mer, mais peu d'études sédimentologiques avaient été menées sur le milieu estuarien proprement dit [1,2]. L'étude sédimentologique du bassin maritime et l'évolution des conditions de navigation dans la partie amont de l'estuaire montrent une augmentation de la sédimentation vaseuse dans les zones exondées et des modifications importantes à l'amont du Port SaintJean, liées à l'évolution du tracé des chenaux [3, 4]. Des photographies aériennes à l'échelle du 1/5000ème ont été réalisées à basse mer sur l'ensemble du bassin en 1953, puis en 1985 à un niveau bas d'environ $4 \mathrm{~m}$ à l'intérieur du bassin de retenue. La comparaison de ces deux vues montre les changements morphologiques intervenus après 20 ans de fonctionnement sous un régime de marée modifié. La surface des zones exondables a été notablement réduite et certains secteurs de berges ont été envasés. Par ailleurs les bancs sableux qui se 
trouvaient à l'embouchure de l'estuaire ont été perturbés, soit par des extractions liées à la construction de l'ouvrage, soit par des érosions liées à l'intensité des courants, particulièrement sur la rive droite proche des pertuis de vannage. A l'inverse, le fond des anses et la partie la plus amont du bassin maritime évoluent vers un colmatage progressif typique de la dynamique sédimentaire estuarienne [5].

La carte sédimentologique de l'estuaire, établie à partir de l'analyse granulométrique d'environ 150 prélèvements de sédiments superficiels, effectués en 1994 dans l'ensemble du bassin maritime, met en évidence les gradients granulométriques longitudinaux et transversaux [6]. A l'embouchure de l'estuaire proche de l'usine, le chenal est tapissé de cailloutis et de sables grossiers, mais le fond des anses est caractérisé par une sédimentation fine de sables vaseux. Au fur et à mesure que l'on va vers l'amont de la retenue, les sédiments deviennent de plus en plus fins, les sables grossiers et moyens faisant place aux sables fins puis à des silts et de la vase pratiquement pure à partir de l'étroiture du Port Saint-Jean / Port Saint-Hubert. La répartition des sédiments superficiels suit la distribution des courants de marée qui sont atténués depuis la mise en service de l'usine, mais qui sont largement guidés par la morphologie de la ria. Les parties resserrées où les courants conservent une certaine vitesse comprennent des sables moyens et grossiers, tandis que les secteurs évasés des anses ou la partie la plus amont du bassin maritime sont des zones d'annulation des courants et il s'y dépose des vases de plus en plus fines. L'évolution granulométrique avec tendance à l'affinement est mise en évidence d'aval en amont de l'estuaire et également depuis le chenal central jusque dans les parties les plus internes et les plus hautes des anses qui scnt colonisées par la végétation halophile.

\section{Analyse sédimentologique de carottages en divers points de l'estuaire.}

Sept carottes courtes $(60 \mathrm{~cm})$ ont été prélevées d'amont en aval du bassin maritime dans les secteurs à sédimentation vaseuse, ainsi qu'à l'extérieur du bassin de retenue et dans un estuaire voisin (1'Arguenon), non aménagé (Fig. 1). A l'intérieur du bassin de retenue, les carottes de Mordreuc et du Chatelier, prélevées en moyenne slikke, sont représentatives de la partie la plus amont de l'estuaire proche de la limite de remontée des eaux marines. Deux autres carottes correspondant à l'aval du bassin maritime ont été prélevées à proximité de l'usine marémotrice, en haute slikke dans l'anse des Rivières et dans un petit affiuent à l'amont de l'ancien moulin à marée de La Richardais. A l'extérieur du 
bassin maritime, le prélèvement de la baie du Prieuré correspond à une sédimentation de moyen estran à l'embouchure de l'estuaire. Enfin, dans l'estuaire voisin de l'Arguenon, la carotte du Guébriand représente un faciès de haute slikke comparable à celui du Chatelier, dans la partie amont d'un petit affiuent, et celle du pont du Guildo a été prélevée dans la partie médiane de l'estuaire.

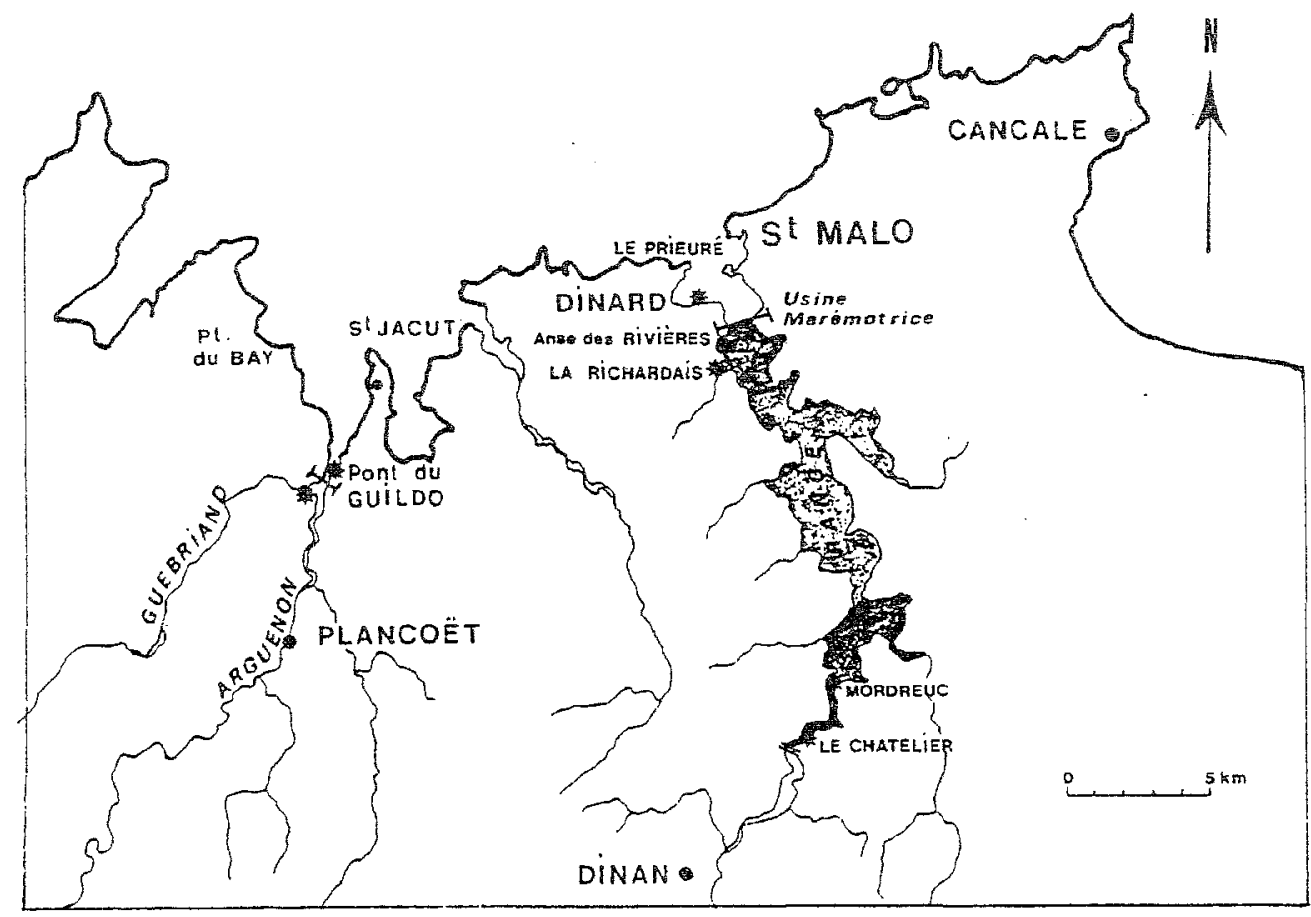

Fig. 1 : Localisation des carottages en Rance et dans l'Arguenon.

\subsection{L'échuse du Chatelier}

Cette carotse est formée d'une vase grise homogène, composée de $70 \%$ de silt $(4-63 \mu \mathrm{m})$ et $25 \%$ d'argile $(<4 \mu \mathrm{m})$ dans sá partie supérieure jusqu'à $45 \mathrm{~cm}$. A partir de cette profondeur, des sables fins $(63-250 \mu \mathrm{m}$ ) apparaissent (environ $25 \%$ ) ainsi que des sables plus grossiers (> 250 $\mu \mathrm{m}$ ) au delà de $50 \mathrm{~cm}$ (Fig. 2). Les associations de foraminifères révèlent une signature oligo-mésohaline, principalement dans l'intervalle compris entre 10 et $40 \mathrm{~cm}$ de profondeur. Entre 40 et $55 \mathrm{~cm}$, un changement de milieu s'observe avec la prédominance de foraminifêres à caractère franchement marin qui se retrouvent également, mais dans de plus faibles proportions dans les $5 \mathrm{~cm}$ superficiels de la carotte. Les associations d'ostracodes présentent une très faible diversité d'espèces entre 0 et $45 \mathrm{~cm}$ de profondeur et les espèces typiquèment marines sont absentes. Au-delà de $45 \mathrm{~cm}$, on observe une plus grande diversité avec des associations 


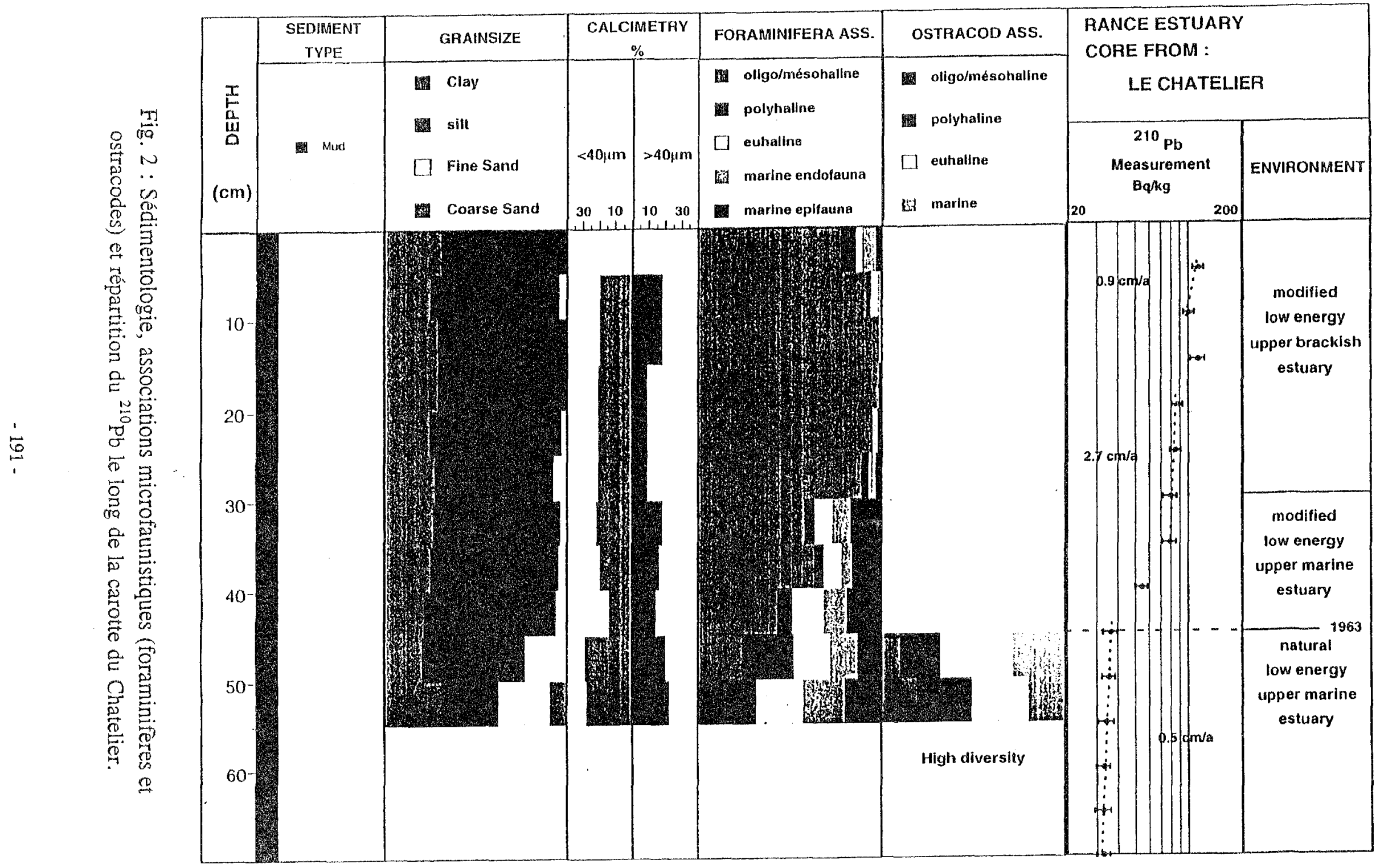


d'ostracodes à caractère marin ou polyhalin. Les mesures de ${ }^{210} \mathrm{~Pb}$ foumissent des taux de sédimentation variables le long de la carotte : entre 60 et $45 \mathrm{~cm}$ de profondeur, les vitesses sont de l'ordre de $0,5 \mathrm{~cm} / a n$, puis un ralentissement important de la sédimentation s'observe entre 45 et $35 \mathrm{~cm}$ où la droite a une pente beaucoup plus faible. A partir de $35 \mathrm{~cm}$ et jusqu'à $15 \mathrm{~cm}$ de profondeur, les taux de sédimentation augmentent rapidement pour atteindre une valeur d'environ $2,7 \mathrm{~cm} /$ an puis, la droite s'infléchit en surface pour se stabiliser à des taux de $0,9 \mathrm{~cm} /$ an [7].

\subsection{Mordreuc}

Le sédiment carotté est une vase homogène composée de $60 \%$ de silt, $25 \%$ d'argile et $12 \%$ de sables fins. Les associations de foraminiferes indiquent une signature essentiellement marine ( 35 à $60 \%$ ), avec une influence estuarienne associée à caractère oligo-mésohalin (10 à $40 \%$ ). Les variations verticales des cortèges de foraminifères révèlent un environnement d'amont d'estuaire de faible énergie avec une influence saumâtre en surface entre 0 et $30 \mathrm{~cm}$ de profondeur et une influence franchement marine en profondeur entre 30 et $58 \mathrm{~cm}$. La microfaune des ostracodes a une origine marine ou euryhaline tout le long de la carotte sauf entre 15 et $30 \mathrm{~cm}$ où les espèces marines sont absentes. Les données du ${ }^{210} \mathrm{~Pb}$ indiquent des taux de sédimentation variables avec une rupture à $30 \mathrm{~cm}$ sous la surface. Les vitesses de sédimentation sont de l'ordre de $0,5 \mathrm{~cm} / \mathrm{an}$ entre 55 et $30 \mathrm{~cm}$ de profondeur et augmentent jusqu'à $1,8 \mathrm{~cm} / \mathrm{an}$ à partir de $30 \mathrm{~cm}$ jusqu'en surface.

\subsection{L'Ange des Rivières}

L'intervalle carotté comprend une alternance de sables fins et grossiers et de lits vaseux. Les foraminifères sont principalement des espèces à caractère estuarien polyhalin (30 à $91 \%$ ), associées à des espèces estuariennes oligo-mésohalines ( 9 à $48 \%$ ). Les variations verticales de la microfaune sont les suivantes :

* de 0 à $15 \mathrm{~cm}$ : environnement d'aval d'estuaire de faible énergie avec des foraminifères allochtones et đes ostracodes marins ;

* de 15 à- $40 \mathrm{~cm}$ : environnement d'aval d'estuaire de forte énergie avec une absence d'ostracodes ;

* de 40 à $50 \mathrm{~cm}$ : environnement estuarien de faible énergie avec présence d'ostracodes essentiellement marins.

Les données du ${ }^{210} \mathrm{~Pb}$ suggèrent une sédimentation non uniforme, des taux de sédimentation ne pouvant être estimés qu'à l'intérieur des différentes couches 
sableuses ou vaseuses. En profondeur les vitesses seraient de l'ordre de $0,16 \mathrm{~cm} /$ an alors qu'en surface jusqu'à $20 \mathrm{~cm}$, elles seraient de $0,3 \mathrm{~cm} / \mathrm{an}$, mais ces discontinuités peuvent être liées aux changements dans la granulométrie du matériel. La partie inférieure de la carotte représente probablement l'environnement de bas estuaire antérieur à la construction de l'usine. La partie moyenne correspond à une augmentation des conditions hydrodynamiques et aux faciès sableux vraisemblablement liés à la réouverture de l'estuaire. Enfin, la partie supérieure est un milieu de bas estuaire de faible énergie où se déposent des sédiments fins dans une zone protégée de fond d'anse.

\subsection{Le Moulin de La Richardais}

La carotte a été prélevée à l'amont d'un petit affluent et est formée d'un sédiment fin homogène composé de $50 \%$ de silt, 10 à $20 \%$ d'argile et 10 à $20 \%$ de sable fin. Les associations de foraminifères révèlent une signature estuarienne essentiellement oligo-haline ( 69 à $90 \%$ ) avec des espèces polyhalines (7-32\%) associées. Les associations à caractère marin et euryhalin sont inférieures à $10 \%$, et il n'y a pas de variation verticale significative. Les cortèges d'ostracodes présentent une très faible diversité (seulement 9 espèces), et sont principalement méso à polyhalins tout le long de la carotte. Les isotopes du $\mathrm{Pb}$ suggèrent une sédimentation assez uniforme, de l'ordre de $0,2 \mathrm{~cm} /$ an en profondeur et de $0,3 \mathrm{~cm} /$ an en surface entre 0 et $15 \mathrm{~cm}$. Ces données indiquent un environnement d'estuaire saumâtre de faible énergie, sans variation évidente avec la profondeur. Le caractère saumâtre du milieu est lié aux apports d'eau douce en provenance du petit affluent de La Richardais .

\subsection{L'anse du Prieuré}

Elle est située à l'embouchure de la Rance, en aval de l'usine marémotrice et le prélèvement est un sable fin (50 à $70 \%$ ) et grossier (20 à 30\%), pauvre en faction fine. Les foraminiferes indiquent principalement une ambiance marine (25 à 50\%) avec une signature euryhaline d'estuaire (15 à 49\%). Les ostracodes sont également des espèces majoritairement marines et euryhalines. Les valeurs des isotopes du $\mathrm{Pb}$ n'ont pu être déterminées en raison de la granulométrie trop grossière du sédiment. La répartition de la microfaune se caractérise par une homogénéité verticale des associations, avec une légère augmentation du caractère oligo-mésohalin dans les derniers $15 \mathrm{~cm}$. Le milieu correspond typiquement un environnement de bas estuaire à caractère marin de forte énergie. 


\subsection{Le pont du Guildo}

Le carottage a été réalisé dans la partie moyenne de l'estuaire de l'Arguenon, et comprend un faciès homogène de silts $(40$ à $60 \%)$ et de sables fins (30 à $40 \%)$. Les foraminifères sont principalement des espèces marines (35 à 56\%) associées à des espèces estuariennes euryhalines (16 à 36\%). Les foraminifères à caractère oligo-mésohalin sont faiblement représentés. Les associations d'ostracodes sont diversifiées et correspondent à un environnement fortement marin ou euryhalin. Les résultats isotopiques indiquent une sédimentation discontinue qui reflète les différentes phases d'érosion et de dépôt liées à la migration latérale des chenaux. Cette carotte est très comparable à celle du Prieuré et correspond à un environnement de bas estuaire essentiellement marin tout le long de la carotte.

\subsection{Le Guébriand.}

Ce petit affluent de la rive gauche de l'Arguenon présente des faciès sédimentaires identiques à ceux de l'écluse du Chatelier dans la Rance,et comporte une vase fine homogène, principalement silteuse (60 à $70 \%)$. Les foraminifêres sont principalement des espèces marines (26 à $53 \%$ ) associées à des espèces à caractère oligo-mésohalin (19 à 45\%). Les associations d'ostracodes reflètent une signature marine et euryhaline, ainsi qu'un environnement oligo-mésohalin lié à la proximité des apports d'eau douce. Les valeurs du ${ }^{210} \mathrm{~Pb}$ sont égales au bruit de fond en profondeur et donnent un taux de sédimentation de $0,24 \mathrm{~cm} /$ an en surface. Cette carotte ne présente pas de variation verticale marquée et a une microfaune mélangée à caractère marin et oligo-mésohalin qui correspond à un milieu estuarien amont de faible énergie.

\section{Conclusion}

L'ensemble des résultats présentés permet de comparer les conditions de sédimentation entre un estuaire modifié, la Rance, et un estuaire naturel, l'Arguenon. Les associations microfaunistiques ont enregistré les modifications de milieu intervenues pendant et après la construction de l'usine marémotrice. La comparaison entre les variations des taux de sédimentation obtenus par les isotopes du $\mathrm{Pb}$ et les changements de salinité reflétés par les associations d'ostracodes et de foraminifères ne fournit pas toujours des résultats homogènes. En particulier, l'existence de remaniements et de discontinuités dans la sédimentation et les variations granulométriques le long des carottes rendent imprécises les déterminations isotopiques et la signature de la microfaune. Cependant, les taux de sédimentation qui étaient inférieurs à 
$1 \mathrm{~cm} / a n$, ont été accélérés dans les parties les plus amont du bassin maritime après la réouverture de l'estuaire.Les nouvelles conditions hydrodynamiques, en particulier la tenue des étales et le relèvement du niveau moyen dans l'estuaire ont modifié la répartition des sédiments, aboutissant à un affinement des vases dans la partie amont et les hautes slikkes des anses du bassin maritime.

\section{Bibliographie}

[1] BERTHOIS L. et C. (1954) (1955) - Etude de la sédimentation dans l'estuaire de la Rance. Première partie : Granulométrie des sédiments. Bull. Lab. Maritime de Dinard. fasc. XL, p. 4-14. Deuxième Partie : Minéralogie des sédiments. Bull. Lab. Maritime de Dinard. fasc. 41, p. 3-18.

[2] BOURCART J. et ROA MORALES P. (1957) - Les dépôts vaseux de la Rance maritime et du Mont Saint-Michel. Bull. Soc. Géol. Fr., (6), VII, p. 545552.

[3] L.C.H.F. (1982) - Etude sédimentologique de l'estuaire de la Rance. Rapport Général Institution Interđépartementale pour l'aménagement de la Rance propre/ DDE Ille et Vilaine. $21 \mathrm{p}$.

[4] L.C.H.F. (1986) - Evolution des conditions de navigation dans la partie amont de l'estuaire de la Rance. Rapport EDF/CRTT Ouest/ GME Bretagne/ DDE Ille et Vilaine. $15 \mathrm{p}$.

[5] BONNOT-COURTOIS C. et LAFOND L.R. (1991) - Caractérisation et comportement des vases dans l'estuaire de la Rance. Rapport EDF/ Lab. de Géomorphologie EPHE. 107 p.

[6] BONNOT-COURTOIS C. CALINE C. LE VOT M. (1995) - A new sedimentological map of the Rance estuary (Brittany, France). IAS-16th Regional Meeting of Sedimentology / Sème Congrès Francais de Sédimentologie ASF. Publication ASF, Paris, $n^{\circ} 22$, p. 24.

[7] BEKS J., BONNOT-COURTOIS C., CALINE B., CHAIT R., CUKALLA A., FERNANDEZ M., LE VOT M., MATHIEU R., OUSSET O. (1995) Sedimentological record of the modified macrotidal regime in the Rance estuary (Brittany, France). IAS-16th Regional Meeting of Sedimentology / Sème Congrès Français de Sédimentologie - ASF. Publication ASF, Paris, $n^{\circ} 22$. p. 15. 